\title{
ALICJA PALETA
}

Università Jagellonica, Cracovia

alicja.paleta@uj.edu.pl

\section{ALCUNE CONSIDERAZIONI SULL'USO DELLA TRADUZIONE NELLA DIDATTICA D'ITALIANO COME LINGUA STRANIERA}

\begin{abstract}
Alicja Paleta, Alcune considerazioni sull'uso della traduzione nella didattica d'italiano come lingua straniera [Some considerations over the use of translation in teaching and learning Italian as a foreign language], Studia Romanica Posnaniensia, Adam Mickiewicz University Press, Poznań, vol. XL/3: 2013, pp. 55-64. ISBN 978-83-232-2638-3. ISSN 0137-2475. eISSN 2084-4158. DOI: 10.7169/ strop2013.403.005
\end{abstract}

The translation, for many years not recommended and even banned in language teaching and learning, lately seems to regain some success at this field. In this article we briefly analyze how the role of this technique has changed throughout the years within the major language teaching theories so as to get to the reasons for its reassessment we can observe nowadays. Finally, we present some considerations on how we can use activities based on translation in the classroom with a particular regard to Italian language teaching to adult learners who are native speakers of Polish.

Keywords: translation, foreign language teaching and learning, Italian language

Nel presente articolo si intende trattare l'argomento della traduzione come tecnica didattica nell'insegnamento delle lingue straniere con un'attenzione particolare verso i discenti di madrelingua polacca che studiano italiano (LS) all'università ${ }^{1}$. Le considerazioni presentate non si possono considerare esaurienti dato che costituiscono il punto di partenza di una ricerca più ampia da completarsi nel quadro di alcuni anni.

\section{LA TRADUZIONE NELL'INSEGNAMENTO DI L2 E LS - PROSPETTIVA DIACRONICA}

L'affermazione da cui spesso si parte parlando della traduzione nell'insegnamento di lingue straniere è che la traduzione è stata sconsigliata come tecnica didattica per l'influsso del metodo grammaticale traduttivo criticato soprattutto perché il discente, lavorando sulla traduzione delle frasi isolate, perdeva il contatto diretto con la lingua

${ }^{1}$ Si pensa soprattutto agli studenti di lingua e letteratura italiana e quelli di lingue e letterature romanze per i quali l'italiano costituisce la seconda lingua romanza. 
appresa che veniva separata dal mittente e dal contesto autentici. Inoltre l'attenzione del processo di insegnamento veniva posta sulla lingua scritta a scapito di quella orale e sull'accuratezza grammaticale a scapito della fluency.

Oggi la valutazione di questo metodo non è più così negativa ed è chiaro che le affermazioni menzionate sopra sono state promosse dagli avversari di questo metodo i quali volevano contestarlo per portare all'approvazione delle loro proposte. La didattica odierna non rigetta più il metodo grammaticale traduttivo nella sua totalità, ma suggerisce piuttosto di integrare consapevolmente il corso con alcune sue tecniche per sviluppare la competenza morfosintattica, il metalinguaggio e le strategie traduttive. Dunque non era l'uso stesso (oppure meglio 'abuso' come riteneva chi era contrario a questo metodo) della traduzione che era sbagliato, ma il ruolo principale che è stato ad essa attribuito.

Le ragioni accademiche dell'abbandono della traduzione sono state formulate nell'ambito delle metodologie che hanno preceduto l'avvento dei metodi diretti. Ci si riferisce al gruppo di fonetisti e linguisti tra cui Wilhelm Viëtor in Germania, Otto Jespersen in Danimarca e Henry Sweet in Inghilterra). Sono stati loro a promuovere il primato della lingua orale sopra quella scritta, del testo completo sopra le frasi isolate, ecc. (Cook, 2010: 4-5). Le loro idee, però, non erano marcate da un estremismo negativo nei confronti della traduzione. Ad esempio nel 1899 Henry Sweet in The practical Study of Languages sosteneva che la traduzione era una tecnica efficace per spiegare il corretto significato delle unità lessicali, mentre escludeva la traduzione dalla L1 in LS ritenendo impossibile tradurre in una lingua che si conosceva solo parzialmente. Otto Jespersen in How to teach a foreign Language del 1904 parlava della traduzione sia in L1 sia in LS. La prima poteva facilitare l'apprendimento del lessico, la seconda doveva aiutare il discente ad acquistare la pratica nella LS. Jespersen sconsigliava, invece, l'uso della traduzione per spiegare i concetti grammaticali della LS.

Con la comparsa dei metodi diretti ${ }^{2}$ si è passati alla totale esclusione della traduzione dall'insegnamento di lingue straniere almeno a livello teorico. L'eliminazione della L1 potrebbe essere, però, spiegata anche da ragioni ben diverse da quelle metodologiche. Per esempio il metodo Berlitz, nato negli Stati Uniti alla fine del XIX secolo, era destinato a numerosi immigrati che erano approdati negli Stati Uniti e che avevano bisogno di comunicare in inglese. Di conseguenza nelle scuole che promuovevano il metodo i gruppi di apprendimento erano plurilingui, gli insegnanti nativi e nel processo di apprendimento si privilegiava la lingua orale sopra quella scritta.

${ }^{2}$ In questa sede ci si riferisce alla definizione dei metodi diretti proposta da Guy Cook secondo il quale si considerano diretti i metodi che escludono totalmente l'uso della L1 degli apprendenti per quanto riguarda sia traduzione sia spiegazione e commento (Cook, 2010: 7). 
Mentre i fonetisti analizzavano l'apprendimento di LS dall'ottica accademica riferendosi all'insegnamento nelle scuole secondarie, i metodi diretti miravano al pubblico adulto che avrebbe dovuto frequentare corsi privati di lingua in tutto il mondo. Per tale motivo questi metodi erano precisamente codificati, gli insegnanti dovevano seguire scrupolosamente le linee guida e i materiali come libri di corso si potevano vendere in tutto il mondo, non essendo indirizzati a un target monolingue. Si può parlare quindi anche delle ragioni economiche del rifiuto della L1 perché le case editrici hanno promosso i metodi diretti, essendo interessate all'incremento della produzione.

Nel periodo successivo alla dominanza dei metodi diretti (più o meno fino agli anni 90) in cui sono sorte diverse ma poco durature proposte didattiche come ad esempio Suggestopaedia, Total Physical Response o Community Language Learning non si può parlare di un esplicito rifiuto della traduzione, ma piuttosto di una tacita ignoranza nella discussione teorica dell'epoca. Gli echi di tale situazione hanno continuato a permanere anche quando sono entrati in vigore gli approcci comunicativi dove il termine 'comunicativo' da molti veniva associato prima di tutto con la lingua orale. Così la traduzione, legata maggiormente alla lettura e alla scrittura, di nuovo non ha meritato un parere favorevole dei teorici anche visto il fatto che il lavoro traduttivo era di solito considerato un lavoro individuale e poco interattivo (Gnutzmann, 2009: 55).

Paradossalmente anche il successo delle prescrizioni del Quadro Comune di Riferimento per le Lingue sembra aver contribuito a ostacolare il ritorno della traduzione fra le tecniche didattiche consigliate o almeno ufficialmente ammesse all'uso in aula. Il Quadro descrive i diversi livelli di riferimento in base alle quattro abilità cioè scrivere, leggere, ascoltare e parlare. La traduzione viene totalmente esclusa dalle attività consigliate dal Quadro per valutare i livelli il che suggerisce indirettamente che fossero giuste le posizioni metodologiche secondo le quali la traduzione andava eliminata dall'insegnamento delle lingue straniere (Leonardi, 2010: 18)³.

\section{LE RAGIONI DELLA RIVALUTAZIONE}

La nascita e lo sviluppo del cognitivismo e dei nuovi studi sull'apprendimento linguistico hanno rivelato che non è possibile eliminare completamente la lingua madre dall'apprendimento di una lingua straniera visto che l'azione del transfer di forme dalla L1 alla L2 scaturisce spontaneamente e inconsapevolmente nella mente del discente $^{4}$. «In effetti ci si rende sempre meglio conto [...] che la lingua materna continua

${ }^{3}$ Bisogna notare però che, anche se bandita dalla metodologia, la traduzione non è mai sparita dalle aule scolastiche perché gli insegnanti vi ricorrevano e finora vi ricorrono ad ogni livello di insegnamento perfino in corsi che si presentano come conformi ai metodi diretti. Per tali motivi Heidi Zojer chiama la traduzione un "amico proibito" dell'insegnante (Zojer, 2009: 32).

${ }^{4}$ Le informazioni più dettagliate sull'attuale approccio verso l'interferenza e il transfert linguistico si possono trovare in: Amati Mehler, Argentieri, Canestri (1990); Salmon, Mariani (2008). 
a permanere a lungo e tenacemente nei discenti che abbordano una nuova lingua e che tale presenza costituisce una sorte di "supersegno" cui vengono continuamente riferite le nuove esperienze linguistiche, una sorta di componente essenziale e duratura del LAD - posto che LAD vi sia - del discente» (Perini, 1982: 239). Dunque una delle ragioni della rivalutazione della traduzione nell'ambito dell'insegnamento delle lingue straniere sembra la convinzione che la traduzione è un'attività innata e naturale e che non si può imparare una LS senza continui riferimenti alla L1 almeno agli inizi di apprendimento 5 .

Vale la pena menzionare anche la relazione fra il ritorno alla traduzione nell'insegnamento delle lingue straniere e lo sviluppo dei translation studies. Il maggiore contributo delle scienze della traduzione pare essere lo spostamento dell'attenzione dalla traduzione intesa come il prodotto finale cioè il testo tradotto verso il processo stesso della traduzione il che permetterebbe di includere nelle tecniche didattiche anche attività che riguardano i processi come l'interpretazione, il code-switching o il code-mixing ${ }^{6}$.

Le fonti di un recente riconoscimento della traduzione sono anche da ritrovare tra i fattori sociali quali globalizzazione, migrazione e il conseguente bilinguismo o multilinguismo che hanno dimostrato chiaramente che la traduzione è un'attività molto vicina alla realtà e non riservata al rigido mondo accademico. "The code-switching and code-mixing - and translation - which arises from such multilingual environments happens in all contexts, from the most personal to the most public, from the inner speech of bilinguals to the world stages of media and politics" (Cook 2009: 44). Di conseguenza la glottodidattica, basandosi sui contributi della sociolinguistica, psicolinguistica e neurolinguistica, sembra oggi concentrarsi su tecniche che si basano su una consapevole accettazione e sfruttamento dei punti di contatto tra le due lingue.

Nonostante un crescente interesse verso l'uso delle L1 dei discenti il quale si è sviluppato verso la fine del XX secolo e nel primo decennio del secolo successivo, la posizione della traduzione nella didattica di LS non è ancora sanzionata e stabile almeno a livello teorico e il lungo silenzio risulta interrotto solo parzialmente. Le proposte metodologiche odierne si concentrano sulla presentazione di argomenti a favore e a scapito dell'uso della traduzione nell'insegnamento di LS.

Gli avversari della reintegrazione della traduzione nella didattica di lingue straniere promuovono le seguenti affermazioni ${ }^{7}$ :

${ }^{5}$ Butzkamm sostiene che la madrelingua costituisce la più importante fonte cognitiva e pedagogica in classe. «The mother tongue opens the door, not only to its own grammar, but to all grammars, inasmuch as it awakes the potential for universal grammar that lies within all of us». Parla di un "monolinguismo illuminato" in cui l'uso della L1 è limitato ma non del tutto escluso (Butzkamm, 2003: 31).

${ }^{6}$ Per approfondire si rimanda a: Bassnett-McGuire (1980), Holmes (1970), Lefevere (1992), Mounin (1963) e (1976), Newmark (1988), Nida (1964), Toury (1980) e (1995), Venuti (1992) e (1995).

${ }^{7}$ Ci si basa sulla rassegna presentata e dettagliatamente analizzata in Zojer (2009: 33-40). 
1) l'uso della traduzione è contrario al principio di monolinguismo in classe;

2) la traduzione, considerata come "la quinta abilità", non è direttamente legata alle altre quattro abilità (ascoltare, parlare, leggere, scrivere) e non dovrebbe essere usata nello sviluppo di nessuna delle macroabilità;

3) la traduzione, se usata in modo eccessivo il che capita spesso e specialmente nella traduzione scritta, porta a trascurare le abilità produttive quali parlare e scrivere;

4) la traduzione è fonte di interferenze a causa del transfer negativo da L1;

5) la traduzione limita la possibilità dell'espressione libera del discente;

6) le attività basate sulla traduzione sono di solito più complesse (perché richiedono il lavoro a più livelli - lessicale, grammaticale, cognitivo, ecc.) e perciò considerate più difficili dalle attività che si concentrano su una sola abilità;

7) la traduzione e la comprensione sono due processi totalmente antagonistici perché la prima non promuove la seconda in quanto allontana la mente dell'apprendente dalla comprensione dirigendola verso le tecniche traduttive;

8) la traduzione in L1 è considerata un'attività del tutto inefficiente dal momento che è lontana dall'obiettivo del corso e cioè dall'apprendimento di LS;

9) la traduzione porta a delle generalizzazioni semantiche inutili e perfino false a livello lessicale perché il significato delle unità lessicali dovrebbe essere appreso insieme alla loro collocazione nel sistema linguistico della LS;

10) la traduzione non dovrebbe essere usata come strumento di valutazione della comprensione del testo in quanto un apprendente può capire il testo e allo stesso tempo non saperlo tradurre.

Dall'altro lato chi è favorevole all'uso della traduzione in classe afferma che:

1) se la traduzione viene usata per approfondire la consapevolezza dei discenti riguardante le differenze tra due lingue diverse, essa può aiutare a eliminare o almeno a limitare il transfer negativo delle strutture della L1 alla LS il che previene le interferenze;

2) le attività basate sulla traduzione involvono e integrano di solito più processi mentali e perciò sono più vicini alla realtà linguistica degli utenti della LS;

3) la traduzione non permette all'apprendente di ricorrere alle strategie di evitamento visto che egli si trova di fronte a un testo/a delle frasi che deve tradurre e non può proporre le proprie alternative (come p. es. in caso di libera produzione scritta);

4) la traduzione è un modo veloce e chiaro di presentare e spiegare unità lessicali nuove che per lo più risponde alla frequente richiesta dei discenti di una rappresentazione semantica in madrelingua il che esclude eventuali non necessari errori semantici;

5) la traduzione può favorire una comprensione dettagliata di un testo da parte dei discenti perché attiva strategie di lettura e comprensione profonde e dettagliate;

6) le attività basate sulla traduzione non sono legate a delle istruzioni difficili oppure alle istruzioni che già in parte contengono le soluzioni del compito che gli apprendenti devono svolgere; 
7) la traduzione di per sé porta alla valutazione della comprensione testuale, sintattica e semantica;

8) l'uso della traduzione in classe influisce positivamente sullo sviluppo della L1 dei discenti ${ }^{8}$;

9) i processi traduttivi arricchiscono la coscienza delle relazioni tra la lingua e la percezione del mondo, della lingua stessa e la cultura;

10) la traduzione aiuta a sviluppare la capacità di usare materiali di sussidio quali dizionari mono- e bilingui, banche dati, ecc.;

11) la traduzione e l'interpretariato possono risultare utili in molte situazioni professionali e private;

12) la traduzione può aiutare gli apprendenti che hanno bisogno di un insegnamento consapevole esplicito e delle regole formulate in modo esplicito (Gnutzmann, 2009: 72).

Se si mettono a confronto le affermazioni appena elencate, si vede che in molti casi abbiamo a che fare con quasi delle "coppie di contrari". Pur essendo queste tesi apparentemente inconciliabili, dopo un'analisi più dettagliata si vede che esse rimandano a diversi punti di vista e si possono conciliare tramite un'applicazione accurata di diverse modalità d'uso che la traduzione può offrire (in L1, in LS; scritta, orale; testi, frasi, unità lessicali; testi già elaborati, testi nuovi, ecc.).

Uno degli argomenti contro l'uso della traduzione che abbiamo appena visto ribadisce che la traduzione non è legata alle quattro abilità e che il suo utilizzo in classe può ostacolare il processo il loro sviluppo. Ma è anche vero che l'elemento che accomuna tutte le attività basate sulla traduzione a diversi livelli di apprendimento è costituito dal rapporto del discente con il testo (in L1 e in LS) e perciò prima di procedere all'attivazione dei processi puramente traduttivi si può cominciare dalle strategie di lettura/ascolto invitando gli apprendenti a lavorare sia sulla comprensione globale (per esempio per definire la difficoltà del testo di partenza) sia sulla comprensione dettagliata (per prepararsi alla traduzione vera e propria, per individuare $i$ frammenti che possano costituire sfide dal punto di vista linguistico o culturale) ${ }^{9}$. Inoltre se si allontana l'attenzione dal processo stesso di tradurre e si punta sulle attività che la precedono, si evita il rischio di frustrare gli apprendenti con un compito troppo complesso $^{10}$.

${ }^{8}$ In caso di studenti universitari di lingue e letterature straniere questo punto diventa particolarmente importante dato che fra i possibili sbocchi professionali gli studenti polacchi (non solo quelli delle specializzazioni in traduttologia) menzionano proprio la traduzione e l'interpretariato.

9 «Any translation task requires many different skills, all of which are important. One skill in particular plays a vital role when a translator first encounters a text, and yet it is usually glossed over in translation studies. This is the reading skill», (Mitchell, 1996: 89).

${ }^{10}$ Per esempio a livelli avanzati $(\mathrm{B} 2-\mathrm{C} 2)$ si può proporre un seguente esercizio: un testo in LS viene diviso in due parti più o meno uguali. Ciascun apprendente riceve solo una parte del testo, la legge e poi la riassume oralmente in LS al compagno che ha letto l'altra parte del testo e poi vice versa. In seguito gli apprendenti si scambiano i fogli con le parti del testo lette prima e ognuno pro- 
Fra le affermazioni presentate sopra, si parlava del fatto che la traduzione è un'attività destinata esclusivamente al lavoro individuale che poi viene corretto e/o commentato in modo frontale. Si potrebbe, però, preparare un breve testo o frasi (a livello lessicale e grammaticale adeguato) in versione bilingue (L1 e LS), ogni discente riceve una delle versioni (in L1 o in LS) e la traduce rispettivamente in LS o L1, alla fine i discenti si mettono in coppie o in piccoli gruppi paragonando le loro versioni. Si conclude con una discussione in plenum sui dubbi sorti durante la discussione. Fra i vantaggi di un tale esercizio, prima di tutto, si può menzionare il fatto che è un'attività da proporre a tutti i livelli di apprendimento. È anche utilizzabile nella fase di controllo nel corso dell'unità didattica perché in tal caso si lavorerà sui testi o sulle frasi con unità lessicali già trattate. Si soddisfa inoltre il criterio della diversificazione delle modalità di lavoro (individuale, a coppie/in gruppi, in plenum).

È vero che se si parla dell'uso della traduzione in classe ci si riferisce soprattutto alla traduzione scritta il che conferma l'affermazione contro il suo uso che si è menzionata prima, però «in realtà anche l'interprete è un traduttore, e la traduzione può essere effettuata, anche in sede didattica, sia partendo dalla lingua scritta che dalla lingua orale» (Calvi, 1984: 457-458). Basta pensare a preparare un testo in LS (una narrazione, una fiaba - dipende dall'obiettivo che si vuole ottenere e dal livello a cui si sta lavorando), dividerlo in due o più parti e, previa lettura della parte assegnata, proporre agli studenti un riassunto orale in L1 ai compagni che hanno letto l'altra parte del testo.

In alcuni casi la L1 sembra perfino indispensabile se vogliamo portare a termine un'attività proposta dai libri di corso i cui autori si dichiarano rappresentanti di approcci in cui l'uso della L1 andrebbe limitato. Si tratta, per esempio, di istruzioni agli esercizi che, visto il livello iniziale a cui appaiono (A1), risultano impossibili da spiegare in un tempo ragionevole senza il ricorso alla L1:

Immaginate di essere una persona importante: trovatevi una nuova identità e scrivete il vostro biglietto da visita. Poi con le domande del punto $22 \mathrm{c}$ scoprite la nuova identità del vicino. Usate la forma di cortesia (Piotti, de Savorgnani 2007: 34).

Che cosa piace al vostro vicino? Provate a indovinare! Aggiungete due cose alla lista, poi mettete le crocette e infine verificate con delle domande come nell'esempio. Ogni preferenza indovinata vale un punto, vince chi indovina di più (Piotti, de Savorgnani 2007: 39).

Anche le attività di comprensione del testo a volte richiedono l'uso della L1 soprattutto per facilitare la spiegazione delle unità lessicali nuove quando la mancata

cede individualmente, attraverso la lettura, a individuare dei brani che sono incomprensibili e/o che secondo loro costituiscono punti difficili per la traduzione (in quanto a: grammatica, lessico, elementi culturali, ecc.). Nel primo caso possono poi in coppie cercare di ricavare il senso dal contesto $\mathrm{e}$, se questo risulta impossibile, ricorrere a dizionari. Nel secondo caso discuteranno sulle possibili strategie da applicare nel corso della traduzione. Si può fare anche una discussione in plenum sul confronto tra i brani individuati dai discenti e sulle proposte di soluzione. Alla fine si procede alla traduzione: individuale a casa oppure sempre in coppie/gruppi in classe. 
conoscenza di queste ultime e l'impossibilità di ricavarne il senso dal contesto ostacola il lavoro e il commento in LS risulta troppo duraturo, se non irrealizzabile. In tal caso, però, sarebbe auspicabile che l'insegnante conoscesse bene il libro di corso con cui lavora e non si limitasse alla mera traduzione in L1 delle parole o espressioni essenziali ma sconosciute, preparando invece delle attività conformi ai principi dell'autonomia e centralità del discente nel processo di apprendimento.

Prima ancora di concludere non si possono tacere le possibili difficoltà che porterebbe con se l'uso della traduzione anche se essa venisse usata in modo programmato e consapevole:

- la traduzione si presta facilmente all'uso ma nei gruppi monolingui dove l'ingegnante conosce la L1 dei discenti;

- gli apprendenti spesso non possiedono un competenza linguistica nella L1 che gli permetta di lavorare liberamente sulla traduzione e discutere sulla qualità della loro traduzione ${ }^{11}$;

- gli apprendenti dovrebbero essere interessati ad un uso di lingua più che strumentale;

- le attività basate sulla traduzione richiedono di solito più lavoro da parte dell'insegnante perché raramente vengono incluse nei libri di corso e la verifica di solito esige più tempo.

Tali questioni non dovrebbero, però, scoraggiare gli insegnanti visto che in cambio avranno a disposizione una vasta gamma di possibili applicazioni per sviluppare la competenza lessicale e grammaticale, la coscienza traduttiva, la coscienza di aspetti contrastivi, per sostenere l'analisi di errori o la revisione del testo, per dimostrare come usare le tecniche e gli strumenti di supporto quali ad esempio dizionari, per sostenere lo sviluppo dell'abilità di scrivere, ecc.

\section{CONCLUSIONE}

Come si è visto non è necessario rifiutare la traduzione, ma si tratta piuttosto di impostare correttamente questa tecnica all'interno del processo di apprendimento, evitando posizioni di estremismo. È vero che se un'attività risulta troppo complessa per apprendenti, può provocare ansia e senso di frustrazione, ma sarà il ruolo dell'in-

${ }^{11}$ Dalla mia prassi come insegnante di italiano come LS a studenti polacchi risulta che di anno in anno è sempre più difficile spiegare alcuni concetti grammaticali servendosi della terminologia linguistica e/o riferendosi a concetti equivalenti o simili del sistema linguistico polacco. A causa delle riforme ministeriali, la linguistica è quasi totalmente sparita dai corsi di lingua e letteratura polacca in scuole elementari e medie e perciò gli studenti del primo ciclo non riescono a mettere a confronto i fenomeni linguistici italiani e polacchi dato che gli mancano le informazioni sulla loro L1. Le tecniche contrastive sono da utilizzare solo dopo alcuni anni di studio, per esempio durante il secondo ciclo, quando avranno già attivato nella mente la cosiddetta "consapevolezza contrastiva". 
segnante scegliere materiale e focalizzare attenzione su degli obiettivi ben determinati per non sovraccaricare i propri studenti.

[...] l'utilizzo di modelli di addestramento definiti "esercizi di traduzione" può divenire fuorviante se non è corredato da motivazioni esplicite, ovvero da un progetto didattico teoricamente coerente. Quando la traduzione viene utilizzata fuori da uno schema teorico argomentato, come accade nella prassi scolastica, viene snaturata, facendosi mezzo innaturale per misurare tutt'altre competenze: in particolare le conoscenze esplicite delle norme grammaticali astratte. In realtà, come dimostrano empiricamente $\mathrm{i}$ bambini bilingui, siamo tutti naturalmente portati a produrre traduzioni pragmatiche funzionali (Salmon, 2005: 245).

In altre parole, non è giusto proporre la traduzione come un metodo didattico autosufficiente senza prendere in considerazione la funzione che dovrebbe svolgere e lo scopo preciso che si vuole raggiungere, né è giusto abolirla totalmente senza porre fiducia nella consapevolezza metodologica dell'insegnante. Inoltre se si tralascia l'ottica secondo cui la traduzione è vista solo come prodotto finale e ci si concentra sul processo stesso della traduzione e sull'intenzione di esprimere lo stesso concetto in due lingue diverse, si apriranno molte opportunità di applicazione della L1 in classe: dal paragone tra L1 e LS attraverso l'uso dei dizionari bilingui fino all'interpretazione dei testi letti in LS e commentati in L1. Queste attività si possono rivelare utili a ogni livello di apprendimento e non solo a livelli avanzati dove i teorici sono più propensi ad ammettere l'uso della L1 e la traduzione in classe. Tutto ciò, però, a patto che si premetta che l'interferenza e il transfer negativo esiste ed è un fatto psicologicamente dato.

Occorre - riconquistata una posizione di sintesi equilibrata - guardarsi dal ritornare su posizioni estreme, ormai almeno teoricamente superate, quali l'analiticismo puro da un lato o il globalismo ingenuo dall'altro. La traduzione può avere una sua funzione, accanto ad altri procedimenti interpretativi ed esercitativi, qualora si ridia al processo di apprendimento linguistico la totalità delle sue dimensioni: tattiche, strategiche, ego-dinamiche. Non c'è dubbio che un retto uso della traduzione possa concorrere ad una acquisizione più cosciente e critica di una lingua, vista non più solo come sistema di abiti e automatismi verbali ma anche, e soprattutto, come sistema cognitivo di strategie di controllo cosciente (Titone, 1984: 53).

\section{BIBLIOGRAFIA}

Amati Mehler, Jacqueline; Argentieri, Simona; CAnestri, Jorge (1990): La Babele dell'inconscio. Lingua madre e lingue straniere nella dimensione psicoanalitica, Milano: Raffaello Cortina Editore.

Bassnett-McGuire, Susan (1980): Translation Studies, London: Methuen.

ButzKamm, Wolfgang (2003): «We Only Learn Language Once. The Role of the Mother Tongue in FL Classrooms: Death of a Dogma», Language Learning Journal, 28/1: 29-39.

Cook, Guy (2010): Translation in Language Teaching: An Argument for Reassessment, Oxford: Oxford University Press.

CALvi, Maria V. (1984): «Il problema della traduzione nella didattica dello spagnolo», in: La traduzione nell'insegnamento delle lingue straniere, Atti del Congresso su "La traduzione nell'in- 
segnamento delle lingue straniere”, Brescia 11-13 aprile 1983, a cura di S. Cigada, Brescia: Editrice La Scuola, 455-472.

Соок, Guy (2010): Translation in Language Teaching: An Argument for Reassessment, Oxford: Oxford University Press.

Gnutzmann, Claus (2009): «Translation as Language Awareness», in: Translation in Second Language Learning and Teaching, a cura di A. Witte, T. Harden, A. Harden, Bern: Peter Lang, 53-77.

Holmes, James S. e altri (a cura di) (1970): The Nature of Translation: Essays in the Theory and Practice of Literary Translation, The Hague: Mouton.

JeSPERSEN, Otto (1904): How to teach a foreign Language, London: George Allen \& Unwin, LTD.

Lefevere, Andre (a cura di) (1992): Translation - History, Culture: a Sourcebook, London: Routledge.

Leonardi, Vanessa (2010): The Role of Translation in Second Language Acquisition. From Theory to Practice, Bern: Peter Lang.

Mitchell, F. (1996): «Reading, an Essential Skill for Professional Translators», in: Teaching Translation in Universities, Present and Future Perspectives, a cura di P. Sewell, I. Higgins, London: Middlesex University Printing Services, 89-102.

Mounin, Georges (1963): Les problèmes théoriques de la traduction, Paris: Gallimard.

Mounin, Georges (1976): Linguistique et traduction, Brussels: Dessartet \& Mardaga.

Newmark, Peter (1988): A Textbook of Translation, New York - London: Prentice Hall.

NIDA, Eugene (1964): Toward a Science of Translating, Leiden: E.J. Brill.

PerINI, Nereo (1982): «Tradurre: una $5^{\circ}$ "abilità" da sviluppare in glottodidattica?», in: Processi traduttivi: teorie ed applicazioni, Atti del Seminario su "La traduzione" Brescia, 19-20 novembre 1981, a cura di S. Cigada, Brescia: Editrice La Scuola, 237-243.

Piotti, Danila; De Savorgnani, Giulia (2007): Universitalia. Corso di italiano. Livello A1-B1, Firenze: Alma Edizioni.

SALmon, Laura (2005): «La didattica della traduzione: dai pregiudizi alle neuroscienze», in: $L a$ traduzione: il paradosso della trasparenza, a cura di A. Guarino, C. Montella, D. Silvestri, M. Vitale, Napoli: Liguori Editore, 241-263.

Salmon, Laura; Mariani, Manuela (2008): Bilinguismo e traduzione. Dalla neurolinguistica alla didattica delle lingue, Milano: FrancoAngeli.

SweEt, Henry (1899): The practical Study of Languages, London: J.M. Dent \& Co.

Tiтone, Renzo (1984): «La traduzione e l'insegnamento delle lingue straniere: problemi psicolinguistici e glottodidattici», in: La traduzione nell'insegnamento delle lingue straniere, Atti del Congresso su "La traduzione nell'insegnamento delle lingue straniere", Brescia, 11-13 aprile 1983, a cura di S. CIGADA, Brescia: Editrice La Scuola, 51-76.

TourY, Gideon (1980): In Search of a Theory of Translation, Tel Aviv: Porter Institute.

Toury, Gideon (1995): Descriptive Translation Studies and Beyond, Amsterdam: John Benjamins.

Venuti, Lawrence (a cura di) (1992): Rethinking Translation: Discourse. Subjectivity. Ideology, London: Routledge.

Venuti, Lawrence (1995): The Translator's Invisibility: A History of Translation. London: Routledge.

ZoJer, Heidi (2009): «The Methodological Potential of translation in Second Language Acquisition: Re-evaluating Translation as a Teaching Tool», in: Translation in Second Language Learning and Teaching, a cura di A. Witte, T. Harden, A. Harden, Bern: Peter Lang, 31-51. 